\title{
The Local Mass Conservation in SPH
}

\author{
Yusuke Imaeda \\ Division of Theoretical Astrophysics, \\ National Astronomical Observatory, Japan \\ Mitaka, Tokyo, 181-8588, Japan \\ Shu-ichiro Inutsuka \\ Department of Physics, Kyoto University \\ Kitashirakawa, Sakyo-ku, Kyoto, 606-8502, Japan
}

\begin{abstract}
Smoothed particle hydrodynamics (SPH) is one of the widely used methods to calculate the various astrophysical fluid dynamics. However, standard SPH cannot accurately describe the long-term evolution of shear flows: The large density error emerges within a dynamical timescale, and the amplitude of the error becomes larger than the value of density itself $(\Delta \rho \gtrsim \rho)$, when we take the mean separation of the particles as the smoothing length. The origin of error is due to the inaccurate description of the continuity equation in the standard SPH formalism. To ensure the local mass conservation property, we have reformulated SPH, in which we distinguish the particle velocity and the fluid velocity for the updation of the particle positions. We find that the present modification provides an accurate description of the density evolution in SPH.
\end{abstract}

\section{Introduction}

Smoothed Particle Hydrodynamics (SPH) is one of the Lagrangian particle methods and is applied to a wide range of the astrophysical flow problem. A broad discussion of the method can be found in a review by Monaghan (1992). However, we demonstrate in this work that the standard SPH method cannot accurately follow the long-term evolution of shear flows. Thus, we modify the $\mathrm{SPH}$ scheme in order to follow the accurate evolution of the shear flows.

\section{Modificaion of SPH}

To demonstrate the drawback of standard SPH, we present a two-dimensional Cartesian shear flow test with constant density. The initial setup is as follows. We set the size of computational domain as $0 \leq x \leq 1$ and $0 \leq y \leq 1$. Initial density is uniform as $\rho=1$ and the shear velocity field is given by $v_{x}=0$ and $v_{y}=\sin (2 \pi x)$. Cyclic boundary conditions are adopted. We set $P=c_{\mathrm{s}}=0$ in this calculation, so that the pressure term vanishes in the equation of motion and the dynamics can be exactly solved. We used $\mathrm{N}=4000$ particles in this 

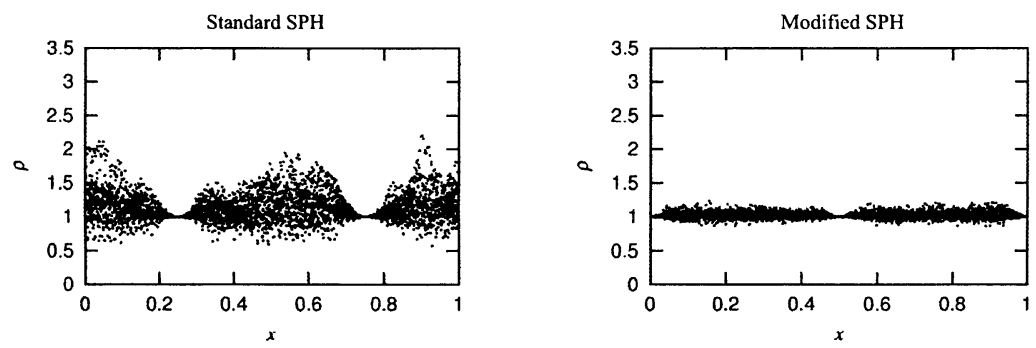

Figure 1. The surface density of Cartesian shear test at the position of each SPH particle after one dynamical time. While the large density errors $(\Delta \rho \gtrsim \rho)$ emerge in the standard SPH result (the left panel), it is reduced considerably in the modified $\mathrm{SPH}$ result (the right panel).

calculation and constant $h=1 / \sqrt{4000}$ was adopted. Although the dynamics is exact, the large density errors $(\Delta \rho \gtrsim \rho)$ emerge after one dynamical time (see the left panel of Fig.1). These errors are originated in the definition of density and the inaccurate solution of continuity equation in standard $\mathrm{SPH}$, which does not ensure the local mass conservation. Thus we compared the Eulerian timederivative of the expression of density in SPH and the continuity equation in standard fluid dynamics and obtained the following relation between the particle velocity $\dot{\boldsymbol{x}}_{\boldsymbol{i}}$ and the fluid velocity $\boldsymbol{v}_{i}$ :

$$
\frac{\sum_{j} m_{j} \dot{\boldsymbol{x}}_{\boldsymbol{j}} W\left(\boldsymbol{x}_{\boldsymbol{j}}-\boldsymbol{x}_{\boldsymbol{i}}\right)}{\sum_{k} m_{k} W\left(\boldsymbol{x}_{\boldsymbol{k}}-\boldsymbol{x}_{\boldsymbol{i}}\right)}=\boldsymbol{v}_{\boldsymbol{i}},
$$

(Inutsuka 2000). Thus $\dot{\boldsymbol{x}}_{\boldsymbol{i}}$ does not coincide with $\boldsymbol{v}_{\boldsymbol{i}}$ in general. In our modified scheme, we determine a set of $\dot{\boldsymbol{x}}_{\boldsymbol{i}}$ from $\mathrm{Eq}$ (1) and use it for the updation of the particle position. The result is shown on the right panel of Fig.1. The detailed procedure of calculation is described in Imaeda \& Inutsuka (2001).

\section{Conclusion}

It is important to ensure the local mass conservation in the SPH scheme when we calculate the long-term evolution of shear flows. We have derived the expression of the particle velocities that ensures the local mass conservation and have used it for the updation of the particle positions, which results in a significant improvement for the density evolution.

\section{References}

Imaeda, Y. \& Inutsuka, S. 2001, submitted to ApJ

Inutsuka, S. 2000, submitted to J. Compt. Phys.

Monaghan, J. J. 1992, ARA\&A, 30, 543 